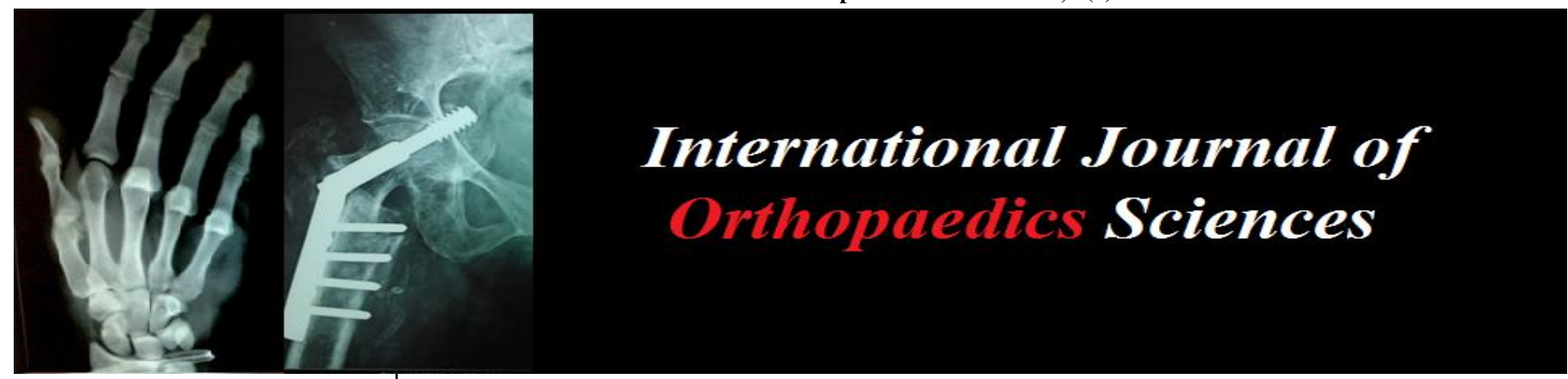

E-ISSN: 2395-1958

P-ISSN: 2706-6630

IJOS 2020; 6(1): 143-146

(C) 2020 IJOS

www.orthopaper.com

Received: 11-11-2019

Accepted: 15-12-2019

\section{Rohan Memon}

Senior Resident, Department of Orthopaedics, NHL Medical College, Ahmedabad, Gujarat, India

Drashtant Patel

Resident, Department of Orthopaedics, NHL Medical College, Ahmedabad, Gujarat, India

Nirav Patel

Resident, Department of Orthopaedics, NHL Medical College, Ahmedabad, Gujarat, India
Corresponding Author:

\section{Rohan Memon}

Senior Resident, Department of Orthopaedics, NHL Medical College, Ahmedabad, Gujarat, India

\section{Functional outcomes of retrograde femoral nailing in extra articular distal third femoral fractures}

\author{
Rohan Memon, Drashtant Patel and Nirav Patel
}

DOI: https://doi.org/10.22271/ortho.2020.v6.i1c.1850

\begin{abstract}
Introduction: The distal third femoral fractures constitute $6 \%$ of all femoral fractures. These fractures pose a great challenge in management due to the involvement of soft tissue injury, intraarticular extension, and injury to the extensor mechanism. The debates on the choice of implant for distal third femoral fractures are on-going among orthopedic surgeons. Controversy remains regarding the optimum device for distal femur fixation.

Objectives: To evaluate and compare the clinical and the radiological outcomes of distal third femoral fractures stabilised using retrograde nailing constructs.

Materials and Methods: This one and half -year prospective observational study includes all patients with fractures of distal femur who underwent retrograde intramedullary interlocking nailing. The patient was placed supine on fracture table with the affected limb flexed to 60o. Through a transpatellar approach, the nail was introduced in a retrograde method after serial reaming. Postoperatively knee range of motion was started immediately and weight-bearing was progressed after signs of fracture union were noted on x-rays. The outcome was evaluated for time taken for fracture union, complications and secondary procedures, knee range of motion and function at 1 year follow up using modified kneerating scale of the hospital for special surgery.

Observations and Results: There were 20 patients who underwent retrograde intramedullary nailing for fractures of distal femur during the study period. According to AO/ASIF system, fractures were classified as $\mathrm{A} 1(\mathrm{n}=15), \mathrm{A} 2(\mathrm{n}=3)$, and A3 $(\mathrm{n}=3)$. In 15 patients, fracture united without complications or secondary procedures. Fractures united at a mean time of 18.4 weeks. There were no varus or valgus malalignments but one patient had significant limb shortening. At the end of 1 year, excellent to good functional outcome was noted in $81 \%$ of patients.

Conclusion: Retrograde distal femoral nailing for AO/ASIF type A and type $\mathrm{C}$ distal third femoral fractures are a proven and reliable method of fixation This safe, successful, reliable and useful technique should find a place in the armamentarium of every orthopedic surgeon dealing with distal third femoral fractures that initially requires attention to details of the technique to prevent complications and to improve the quality of life.
\end{abstract}

Keywords: Distal third femoral fractures, retrograde nail, locking compression plate

\section{Introduction}

The incidences of distal third femoral fractures are 37 per 1 lakh person-years. The distal third femoral fractures constitute $6 \%$ of all femoral fractures ${ }^{[1]}$. These fractures occur in bi-modal distributionn as high energy trauma in younger age group and low energy fractures due to osteoporosis in the elder age group. Due to extensive soft tissue damage, an extension of the fracture into the knee joint, neurovascular damage and injury to the extensor mechanism in the distal femur, these distal third femoral injuries were complicated and difficult to manage ${ }^{[2]}$. The orthodox management of distal third femur fractures described by Watson Jones and John Charnley comprised of skeletal traction, manipulationn of fracture and external immobilisation in the form of casts and braces. This method was associated with deformity, shortening, fibrous ankylosis of the knee, knee joint incongruity, malunion, quadriceps wasting, knee instability, and early knee osteoarthritis. The complications with these treatment methods led to the development of newer techniques of fracture management. The distal third femoral fractures are best treated with open/closed reduction and surgical stabilisation with load sharing or load-bearing implants ${ }^{[3]}$. 
Most surgeons agree that distal third femoral fractures need to be treated operatively to achieve optimal outcomes. The debate continues around the choice of implant for fixation of metaphyseal-diaphyseal fractures. The type of device depends on variables, fracture personality, and soft tissues. The current method of open reduction and internal fixation has become evident in the recent years with good results being obtained with the AO blade plate, dynamic condylar screw, intramedullary supracondylar nail and locking compression plate $^{[4,5]}$.

The intramedullary nailing act as a load sharing device which gives good results with minimal soft tissue dissection and good stability. The advantage of an intramedullary device is that it aligns the femoral shaft with condyles reducing the tendency to place various movements at the fracture site. The reduced bending movement of an intramedullary device has reduced failure of fixation in osteoporotic bone ${ }^{[6]}$. Intramedullary nails offer potential biomechanical advantages over plates and screws because their intramedullary location results in less stress on the implant, they have the potential for load sharing and can be inserted with minimal stripping of soft tissues The retrograde intramedullary distal femoral nail has the advantages of fracture hematoma preservation reduced blood loss, minimal soft tissue damage, less operative time and reduced rate of infection ${ }^{[7]}$.

\section{Objectives}

To evaluate and compare the clinical and the radiological outcomes of distal third femoral fractures stabilised using retrograde nailing constructs.

\section{Materials and Methods}

In this one and half year prospective study, 20 patients who underwent retrograde interlocking nailing for fractures of distal femur in a tertiary care center were included. Informed written consent was taken from all patients. Institutional ethical clearance was obtained for this study.

\section{Inclusion criteria}

- Patients with age between 20 to 70 years of age

- Patients with clinically and radiologically confirmed distal third femoral fractures of AO types A1, A2, A3, $\mathrm{C} 1$, and $\mathrm{C} 2$

- Patients with type $1,2,3 \mathrm{a}$ and $3 \mathrm{~b}$ compound fractures according to Gustilo Anderson classification for open fractures

- $\quad$ patients who are medically fit for surgery

- patients who have given written consent for treatment as per our protocol

- Exclusion criteria

- patient with age less than 20 and more than 70 years of age

- Patients with proximal third and middle third femoral fractures

- $\quad$ Patients with distal third femoral fractures of AO types B and $\mathrm{C} 3$

- Patients with type 3C compound femoral fractures according to Gustilo Anderson classification for open fractures

- Patients with pathological distal third femoral fractures

- Patients who are medically unfit for surgery

- Patients who has not given written consent for treatment as per our protocol

The patient was placed on a radiolucent table in supine position with the affected limb flexed at 60 Transpatellar approach to the knee was obtained and the insertion point at the intercondylar notch anterior to Blumensaat's line along the femoral shaft axis was marked using image intensifier. Guide wire was passed through the proximal fragment after reduction and medullary canal was reamed in $0.5 \mathrm{~mm}$ increments until cortical contact appreciated. All fractures were reduced by closed methods. All fractures were statically locked.
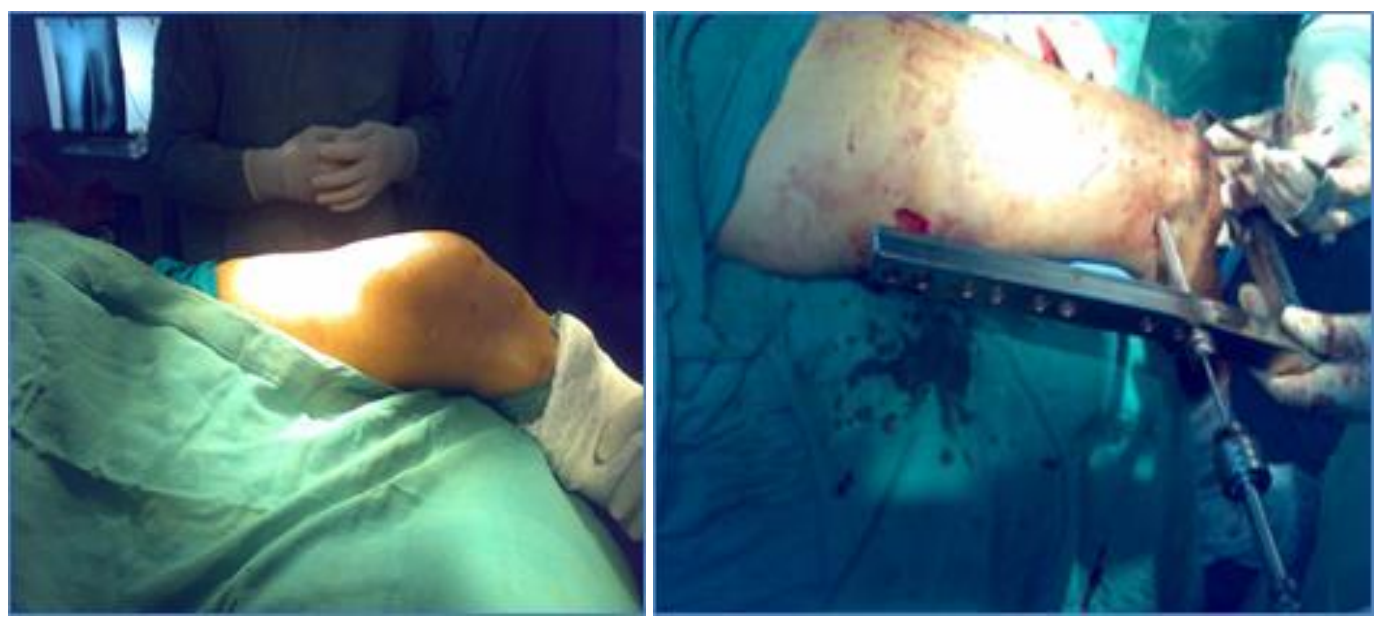

Fig 1: Intraoperative images

\section{Postoperative protocol}

Postoperatively, range of motion exercises were initiated in 2 days. Non weight-bearing mobilization was allowed immediately and gradually promoted to full weight bearing as bridging callus was noted on x-rays at follow-ups. Patients were followed up at 6 weeks, 3 months, 6 months and 1 year. Patients were followed with $\mathrm{x}$-rays and Modified Knee-Rating Scale of Hospital for special surgery for assessing the functional outcome. 

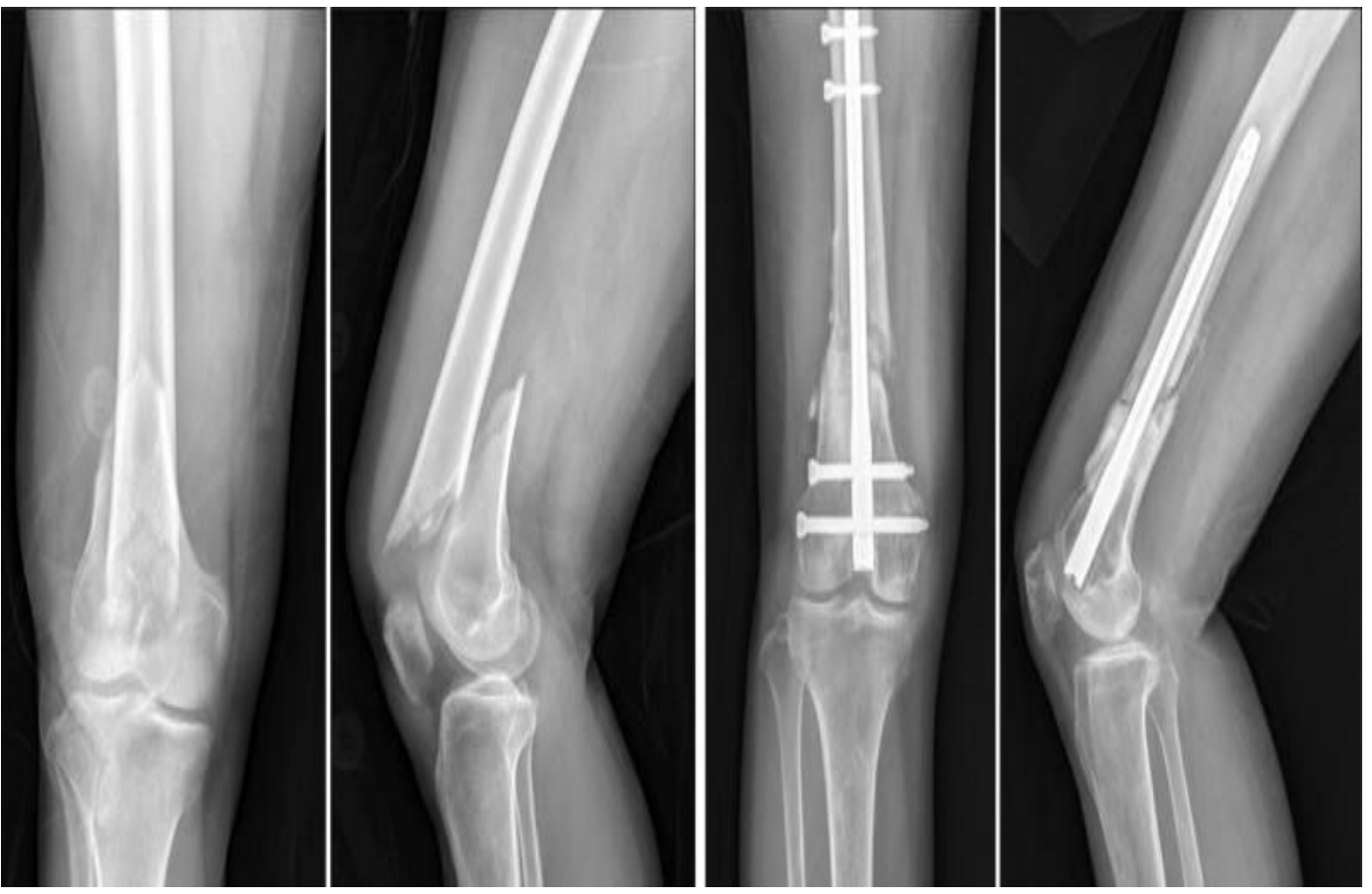

Fig 2: Preoperative and postoperative xray images

\section{Results}

There were 15 males and 5 females aged from 24 to 77 years (mean 50 years) in the study. Right femur was involved in 14 subjects and left in 6. Road traffic accident was the commonest mode of injury followed by fall from height and trivial injury. There were 18 closed and 2 open fractures. Average operative time was 1 hour 48 minutes. Fracture union was noted in 17 patients without any secondary procedures or complications. Mean time for union was 18.4 weeks (SD 2.7 weeks). Of these, 3 patients underwent dynamization because of features of delayed union. All of these fractures united after dynamization. No patients developed non-union. At the end of 1 year Modified KneeRating Scale showed $81 \%$ excellent to good scores for pain and function as given in Figure 1. There were no significant varus/valgus mal-alignments.

Table 1: Showing observations in tabular form

\begin{tabular}{|c|c|}
\hline Mean time for radiological union & 18.4 weeks \\
\hline Varus /valgus alignment & none \\
\hline & $>120-15$ \\
Knee range of movement & $<0-120-3$ \\
& $390-1$ \\
\hline Delayed union & none \\
\hline Non union & 1 \\
\hline Shortening &
\end{tabular}

\section{Discussion}

Intramedullary nailing and bridge plating are the frequently used treatment modalities for fractures of distal femur ${ }^{[8]}$. They are biologic methods of fixation with less soft-tissue dissection and devitalization. As the fracture hematoma is preserved in these methods of fixation, complications such as nonunion and infection are less compared to traditional methods of open reduction and plating. Compared to plating, intramedullary nails are load sharing devices allowing early weight-bearing postoperatively. Retrograde intramedullary nailing has many advantages over the antegrade method of fixation for these fractures. Polytrauma, ipsilateral pelvic, acetabular, tibial or femoral neck fractures and hip dislocations, ipsilateral hip implants, obesity and pregnancy are the clinical conditions where RIMN is preferred to AIMN [9]. However, limitations of RIMN include interference with the knee function and longer union time in case of lower third femoral fractures.

The average operating time in our study was 1 hour 48 minutes which was less than the time required for antegrade nailing of distal femur fractures ${ }^{[10]}$. However, the mean time for fracture union was less than that for bridge plating which could be due to preservation of fracture hematoma and early postoperative weight bearing in retrograde nailing ${ }^{[11]}$ Average time taken for union in open fractures was 21.3 weeks which was comparable with the previous studies ${ }^{[12]}$. In our study, 3 patients $(14.3 \%)$ who had fractures at the middle-distal third junction had features of delayed union and underwent dynamization as a secondary procedure. Following dynamization, these fractures united uneventfully. This is comparable to the reports in the previous studies ${ }^{[14]}$. One complication which was noted in our study was the stress fracture at the tip of the nail. This could be due to the exaggerated anterolateral bowing of femur in osteoporotic bone which causes stress rising at the proximal fixation point. Knee pain, stiffness and function postoperatively have been the major concerns following RIMN. Various studies have shown that RIMN does not significantly affect the range of movement of the knee ${ }^{[15]}$. In our study, $85.7 \%$ patients achieved $>110 \mathrm{o}$ flexion at the end of 1 year follow-up. Modified kneerating scale of Hospital for Special Surgery at the end of 1 year showed good to excellent results in $81 \%$ patients which are comparable to previously reported studies. [16].

\section{Conclusion}

Retrograde intramedullary nail fixation is an effective method for treating fractures of distal femur. It provides a stable intramedullary fixation with less soft tissue injury. This method does not interfere significantly with the knee function postoperatively with negligible complications. 


\section{References}

1. Court-Brown CM, Caesar B. Epidemiology of adult fractures: A review. Injury. 2006; 37:691-697.

2. Kolmert L, Wulff K. Epidemiology and treatment of distal femoral fractures in adults. Acta Orthop Scand. 1982; 53:957-962.

3. Wilson JN. Watson Jone's: Fractures and joint injuries. (6 $6^{\text {th }}$ edn $), 1982,1003-1070$.

4. Lupescu O, Nagea M, Patru C, Vasilache C, Popescu GI, et al. Treatment options for distal femoral fractures. Maedica J Clin Med. 2015; 10:117-122.

5. Hartin NL, Harris I, Kaushik H. Retrograde nailing versus fixed angled blade plating for supra-condylar femoral fractures: A randomized controlled trial. ANZ J Surg. 2006; 76:290-294.

6. Ehlinger M, Ducrot G, Adam P, Bonnomet F, Jacob M et al. Distal femur fractures. Surgical techniques and a review of the literature. Ortho Traum Surg Res. 2013; 99:353-360.

7. Salem KH, Maier D, Keppler P, Kinzl L, Gebhard F. Limb malalignment and functional outcome after antegrade versus retrograde intramedullary nailing in distal femoral fractures. J Trauma. 2006; 61:375-381.

8. Patterson BM, Routt ML Jr, Bernischke SK, Hansen ST Jr. Retrograde nailing of femoral shaft fractures. J Trauma. 1995; 38:38-43.

9. Kulkarni SG, Varshneya A, Kulkarni GS, Kulkarni MG, Kulkarni VS, Kulkarni RM. Antegrade Interlocking nailing for distal femoral fractures. J Ortho Surg. 2012; 20(1):48-54.

10. Moed BR, Watson JT. Retrograde intramedullary nailing, without reaming, of fractures of femoral shaft in multiply injured patients. J Bone Joint Surg Am. 1995; 77:1520-7.

11. Demirta AS, Azboy I, Gem M, Alemdar C. Comparison of retrograde intramedullary nailing and bridge plating in the treatment of extra-articular fractures of distal femur. Acta Orthop Traumatol Turc. 2014; 48(5):521-6.

12. Henderson CE, Lujan T, Bottlang M, Fitzpatrick DC, Madey SM, Marsh JL. Stabilization of distal femur fractures with intramedullary nails and locking plates: differences in callus formation. Iowa Orthop J. 2010; 30:61-8.

13. Surulivel VJ, Ganesan GR, Rajasekharan R. Dynamic condylar screw versus supracondylar nail in the management of supracondylar fracture distal femur. Int Surg J. 2015; 2(3):373-6.

14. Tornetta P, Tiburzi D. Antegrade or retrograde femoral nailing. A prospective randomized trial. J Bone Joint Surg Br. 2000; 82:652-4.

15. Gupta SKV, Govindappa CVS, Yalamanchili RK. Outcome of retrograde intramedullary nailing and locking compression plating of distal femoral fractures in adults. OA Orthopaedics. 2013; 1:23.

16. Thompson AB, Driver R, Kregor PJ, Obremskey WT. Longterm functional outcomes after intraarticular distal femur fractures: ORIF versus retrograde intramedullary nailing. Orthopedics. 2008; 31:748-750. 